\title{
The value of chronic suppressive therapy with itraconazole versus clotrimazole in women with recurrent vaginal candidiasis
}

\author{
I W Fong
}

\begin{abstract}
Objective-To determine the comparative efficacy of oral itraconazole versus intravaginal clotrimazole in suppressing recurrent episodes of vulvovaginal candidiasis.

Design-Prospective randomised open study of women with recurrent vulvovaginal candidiasis. Clinical and microbiological assessments were made monthly for 12 months.

Setting-Women's Clinic of a University teaching hospital.

Subjects-Forty-four otherwise healthy, non-pregnant women, with at least four proven episodes of candida vaginitis in the last year were enrolled into the study.

Intervention-After an acute episode of candida vaginitis, 22 women received oral itraconazole $200 \mathrm{mg}$ daily for five days, then $200 \mathrm{mg}$ twice weekly for six months; and 22 women received intra-vaginal clotrimazole $200 \mathrm{mg}$ ovules daily for five days, then $200 \mathrm{mg}$ twice weekly for six months.
\end{abstract}

Main Outcome Measures-Symptomatic recurrent clinical vulvovaginal candidiasis during the first six months of suppressive therapy was the major endpoint. A secondary endpoint was recurrent candida vaginitis within six months after completion of therapy.

Results-Six patients did not complete the study, one in the itraconazole group and five in the clotrimazole group. Of the evaluable patients, seven of 21 patients $(33.3 \%)$ in the itraconazole group versus none $(0 \%)$ of 17 patients on clotrimazole were failures on suppressive therapy, $\mathbf{p}=0 \cdot 02$. Following discontinuation of suppressive therapy, recurrences of candida vaginitis were similar, $10(47 \cdot 6 \%)$ of patients on itraconazole ( $95 \%$ confidence interval (CI) $27-67 \%$ ), versus $11(64 \%)$ patients on clotrimazole (CI $41-87 \%$ ), $\mathbf{p}=0 \cdot 15$.

Conclusion-Intermittent suppressive therapy with clotrimazole was more effective than itraconazole in preventing recurrent candida vaginitis, provided patients adhered to the regimen. Recurrence of vaginitis was common with both regimens after stopping suppressive therapy.

(Genitourin Med 1992;68:374-377)

Introduction

A small group of women with frequently recurring and persistent vulvovaginal candidiasis continues to be a therapeutic challenge to family physicians and gynaecologists. More importantly, the chronic symptoms associated with recurrent vulvovaginal candidiasis including pruritis, vaginal discharge, vaginal discomfort and soreness and dyspareunia result in severe strain on sexual relationships. The aetiology of recurrent infection is usually unknown' but several predisposing factors have been suggested, such as impaired cellular immunity $^{2}$ re-infection from the sexual partner, ${ }^{3}$ and re-infection from the intestines. ${ }^{4}$

The therapeutic management of recurrent candidal vaginitis has been attempted by both the patients and their physician without success. Homeopathic remedies, diets, topical antifungal medications, intra-vaginal yogurt, and oral lactobacilli have been used without any permanent cure. ${ }^{1}$

Chronic suppressive therapy using cyclical or daily continuous therapy with topical or oral antifungal agents ${ }^{56}$ have been used to control or suppress recurrences with success. The rate of symptomatic candidal vaginitis was reduced while long term therapy was provided but the infection recurred following the cessation of therapy.

The advantage of intermittent suppressive therapy over continuous daily therapy seems obvious. Less use of medication reduces the risk of side effects and the overall cost. This study was designed to compare two forms of intermittent suppressive therapy: (1) Clotrimazole administered intra-vaginally and (2) Itraconazole administered orally. Itraconazole is a new investigational, systemically absorbed antifungal agent which has shown promising results in animal models and clinical studies for systemic and mucosal fungal infections, including candidiasis. ${ }^{78}$ Animal studies of experimental vaginal candidosis ${ }^{9}$ have indicated that itraconazole is three to five times more potent than ketoconazole. Moreover, clinical studies have shown itraconazole to be an effective treatment of candida vaginitis as a single day treatment. ${ }^{10}$

\section{Method}

A prospective randomised study was conducted among 44 women with recurrent, clinical symptomatic vaginal candidiasis; defined as greater than four episodes (proven by culture) in the past year. Women with no chronic underlying illness, including diabetes mellitus, chronic use of antibiotics, nor steroids (or known immunodeficiency) were selected for the study. Patients were random-
This study was presented in part at the 30th ICAAC Atlanta, GA, 21
October, 1990. 
ised after an acute episode of candidiasis to receive:

A. Itraconazole $200 \mathrm{mg}$ orally daily (administered as $100 \mathrm{mg}$ twice daily with meals) for 5 days, then $200 \mathrm{mg}$ twice weekly for 6 months, or

B. Clotrimazole vaginal ovules $200 \mathrm{mg}$ (Canesten 3) daily for 5 days, then $200 \mathrm{mg}$ twice weekly for 6 months.

The random numbers were computer generated. Numbered, sealed envelopes containing the therapeutic regimen were kept in the Pharmacy Department ensuring that the investigator remained blinded to the randomisation.

Diagnosis of candida vaginitis was established by microbiological and clinical criteria. A speculum examination of the vagina was done on all patients, noting the presence or absence of inflammation; excessive or normal discharge; appearance and odour of discharge. Patients were enrolled into the study, after an acute episode of vulvovaginal candidiasis.

A clinical assessment was conducted at 1-2 weeks after initial treatment and then monthly for one year. Vaginal washings (obtained by irritation with $5 \mathrm{ml}$ of sterile saline) and swabs from the vulva and vagina were cultured for yeast on Sabouraud's and blood agar containing antibiotics to suppress normal bacterial flora. Identification of yeasts was done by the germ tube method using human serum, production of chlamydospores on Tween 90 and sugar assimilation when necessary. Initial genital swabs were cultured for Neisseria gonorrhoea, Gardnarella vaginalis, Chlamydia trachomatis, and Trichomonas vaginalis. Blood tests including complete blood count, differential, white cell count, platelet count, blood sugar, serum creatinine, serum glutamic oxaloacetic transaminase (SGOT), alkaline phosphatase bilirubin and pregnancy tests were completed initially, once monthly at each visit for 6 months and on completion of the course of therapy.

All patients were assessed for efficacy, safety, tolerance, and compliance with therapy. Patients were monitored for 6 months following discontinuation of suppressive therapy for the recurrence of candidiasis. A recurrence was considered definite if cultures were poistive for $C$ albicans and associated with signs or symptoms of candidiasis. Clinical symptomatic vaginal candidiasis was definite in patients with symptoms of any of the following: excessive vaginal discharge, itching, burning, dysuria or dyspareunia, associated with clinical findings of white or creamy vaginal discharge in excessive amount, with or without redness of vulva or vagina, and with positive culture or smear of $C$ albicans.

Asymptomatic candida infection or colonisation was in patients without any complaints but vaginal culture positive for candida. Cure was the disappearance of all symptoms and signs of clinical candida infection with negative vaginal culture for candida posttherapy for up to two months. Failure or persistence of infection-no significant, or minimal improvement of symptoms and signs with continued recovery of candida.

This study was approved by the Clinical Trials Committee of St Michael's Hospital and written informed consent was obtained from all patients prior to enrolment.

\section{Statistical analysis}

The data were analysed by two methods: (1) only patients adhering to the protocol were analysed for efficacy; patients lost to follow-up or non-compliant with the treatment regimen were excluded from analysis; (2) intent to treat analysis (all patients were analysed irrespective of compliance); patients lost to follow up or had recurrences due to non-compliance were assessed as failures. For continuous variables the groups were compared using Student's $t$ test. For categorical variables, the groups were compared by Chi-square analysis or Fisher's exact test (if the number in any cell was less than five). Ninety-five percent confidence intervals (CI) were calculated for all results expressed as rates.

\section{Results}

Forty-four patients were enrolled into the study with 22 patients in each group. A comparison of the demographic features (table 1) shows that the two groups were similar. None of the following were significantly different: the mean age; the use of oral contraceptives; post menopausal state; underlying illness; and the mean number of episodes of vaginitis per year.

All the candida isolates recovered during study were identified as $C$ albicans, except for three strains which were Candida non-albicans species (not further identified). One of the isolates was recovered at enrollment and two on follow-up visits, all in the intraconazole group and associated with symptoms of vaginitis.

Six patients did not complete the study; one in the itraconazole group, because of her desire to become pregnant, and five in the clotrimazole group. Two of the five patients were non-compliant with the medications and three were lost to follow up after 1-4 months. Thus, the fully evaluable patients included 21 patients in the itraconazole group and 17 patients in the clotrimazole group. Of these evaluable patients two $(9.5 \%)$ receiving itraconazole had persistence of symptoms versus none in the cotrimazole group. Five other patients $(23.8 \%)$ had recurrence while on suppressive therapy with itraconazole (CI

Table 1 Demographic features of women with recurrent vaginitis

\begin{tabular}{lll}
\hline Treatment & Itraconazole & Clotrimazole \\
\hline No of patients & 22 & 22 \\
Mean age, SD $(\mathrm{yrs})$ & $33 \cdot 6,7 \cdot 4$ & $31,5 \cdot 6$ \\
Range (yrs) & $23-49$ & $19-42$ \\
BCP use & $5(23 \%)$ & $7(32 \%)$ \\
Post menopausal & 1 & 1 \\
$\begin{array}{l}\text { Mean no candidiasis episodes } \\
\text { per year, SD }\end{array}$ & $8 \cdot 8,2 \cdot 8$ & $9 \cdot 7,2 \cdot 8$ \\
\hline BCP $=$ Birth control pill & &
\end{tabular}

$\mathrm{BCP}=$ Birth control pill
$\mathrm{SD}=$ Standard deviation 
Table 2 Recurrence of candida vaginitis during and after suppressive therapy

\begin{tabular}{lccc}
\hline & Itraconazole & Clotrimazole & $p$ Value \\
\hline No enrolled & 22 & 22 & - \\
No completing study & 21 & 17 & $0 \cdot 08$ \\
Persistence of symptoms & 2 & 0 & $0 \cdot 24$ \\
Recurrence during therapy & 5 & 0 & $0 \cdot 04$ \\
Recurrence after therapy & 10 & 11 & $0 \cdot 15$ \\
\hline
\end{tabular}

$12-43 \%)$ versus none $(0 \%)$ in the clotrimazole group (CI $0-8 \%$ ). Thus, seven patients $(33.3 \%)$ on itraconazole versus $0(0 \%)$ on clotrimazole were failures on suppressive therapy, $p=0.02$. The number of patients with asymptomatic colonisation with candida during suppressive therapy were $3(14.3 \%)$ in the itraconazole group, and $4(23.5 \%)$ in the clotrimazole group (not statistically significant).

Following discontinuation of chronic suppressive therapy, the recurrence rate in the subsequent six months were high in both groups. Ten $(47.6 \%)$ of the patients in the itraconazole group (CI 27-67\%) had recurrences of candidiasis, versus 11 patients $(64 \%)$ in the clotrimazole group (CI 41-87\%), p $>0 \cdot 15$ (table 2).

Most of the recurrences in both groups occurred within three months of stopping the suppressive therapy. Thus, after 12 months total follow up only four patients $(19 \%)$ in the itraconazole group $(\mathrm{CI}=2-36 \%)$ and six patients $(35.3 \%)$ in the clotrimazole $(\mathrm{CI}=12-58 \%)$ remained free of symptomatic candida vulvovaginitis, $\mathrm{p}=0 \cdot 16$.

With an intent to treat analysis, the results are somewhat different, especially for the first six months while on chronic suppressive treatment. The failure rate on suppressive treatment in the itraconazole group was eight of 22 patients $(36.4 \%), C I=16-56 \%$; and the failure rate in the clotrimazole group was five of 22 patients $(22 \cdot 7 \%), \mathrm{CI}=5-41 \%$, $\mathrm{p}=0 \cdot 16$.

Mild adverse events occurred in seven women $(31.8 \%)$ treated with itraconazole (CI $21-55 \%)$ versus $0(0 \%)$ in the clotrimazole group $(\mathrm{CI}=0-8 \%), \mathrm{p}=0.02$. These side effects included mild nausea (4 patients), mild diarrhoea ( 1 patient), headaches ( 1 patient) and transient faintness with dizziness (1 patient). None of the patients required discontinuation of study medication because of adverse events.

\section{Discussion}

The management of patients with recurrent vulvovaginal candidiasis has been difficult and frustrating for both patients and physicians. Presently, there is no known long lasting cure and only a few studies have addressed the issue of suppressive therapy to control the patients' symptoms.

Davidson and Mould ${ }^{5}$ were the first to use intermittent suppressive therapy with clotrimazole (cream and pessaries) on the 5th to 11 th day of the menstrual cycle for four months. In comparison with placebo clotrimazole was highly effective in reducing symptomatic dis- ease $(1 / 18$ versus $10 / 19, p<0.01)$. However, clotrimazole did not prevent recurrent candida colonisation and recurrence were common after cessation of suppressive therapy.

In another controlled study Sobel ${ }^{6}$ randomised 74 women with recurrent vulvovaginal candidiasis to receive placebo, ketoconazole $400 \mathrm{mg}$ daily for five days at the onset of menses for six months, or low dose ketoconazole $100 \mathrm{mg}$ daily for six months. During the period of suppressive therapy, symptomatic recurrence of candida vaginitis occurred in 15 of 21 women $(71.4 \%)$ treated with placebo, in six of $21(28.6 \%)$ and one of $21(4.8 \%)$ women on intermittent and daily ketoconazole respectively. After the prophylaxis was discontinued the recurrence rates were high in both treated groups.

In this study, intermittent (twice weekly) therapy with oral itraconazole or intravaginal clotrimazole appear to be effective in reducing symptomatic recurrence of candida vaginitis when compared with the results with placebo obtained in other studies. ${ }^{56}$ The recurrence rate with twice weekly itraconazole $(23.8 \%)$ is similar to that of cyclical monthly five day courses of ketoconazole $(28.6 \%)$. However, twice weekly clotrimazole intravaginally is more effective (if compliance is good) and appears to be similar in suppressive value as daily oral ketoconazole (recurrence rates $0 \%$ versus $4 \cdot 8 \%$, respectively).

The reason for the greater efficacy of clotrimazole over itraconazole may be related to a higher and more persistent concentration of active antifungal drug in the vagina. Recent pharmacokinetic studies have shown that itraconazole is variably absorbed, with wide intersubject variation in serum concentration and thus, tissue levels may not be very predictable. ${ }^{11}$ Although the total itraconazole concentration in vaginal tissue has been found to be higher than blood levels, ${ }^{12}$ the free (active) drug concentration in tissue fluid is much lower than the free serum concentration. ${ }^{13}$

Although itraconazole was associated with a higher prevalence of side effects compared to clotrimazole, these were mild and well tolerated. In fact compliance was greater with itraconazole, probably because of the convenience of an oral administration. Thus, on balance (as shown by the intent to treat analysis) itraconazole and clotrimazole overall may have comparable efficacy. However, because of the relatively small sample size the power of this observation is not very high and the beta error would be significant.

The high prevalence of recurrence of candida vaginitis after discontinuation of suppressive treatment in our study is similar to the findings of Sobel, ${ }^{6}$ and Davidson and Mould. However, a small number of women will remain free of symptoms for six months or more after discontinuation of chronic suppressive therapy.

The choice of an agent for chronic suppressive therapy must be assessed in terms of efficacy, toxicity, compliance and cost. Both daily ketoconazole and intermittent intravaginal clotrimazole are highly effective. How- 
ever, there is a greater potential for systemic toxicity with ketoconazole, especially hepatotoxicity. Hepatitis has been associated with ketoconazole, and the risk of liver injury has been estimated at one in 15,000 people, especially for women over 50 years of age. ${ }^{614}$ Clotrimazole may be associated with local discomfort but no systemic toxicity.

Although intravaginal administration of a medication appears to be less acceptable than oral administration, daily administration may be associated with less compliance than twice weekly administrations. Finally the cost of a six months supply of ketoconazole $100 \mathrm{mg}$ daily (as recommended by Sobel, ${ }^{6}$ ) is $\$ 170.00$ versus the cost of clotrimazole $200 \mathrm{mg}$ (Canesten 3 ), twice weekly is $\$ 228.00$ (in Canada).

I am grateful to Drs P McCleary and M Simbul, Ms A Carvalho and to Mrs D Bajhan for their assistance in preparation of this manuscript. This study was supported by a grant from Janssen Pharmaceutica Inc, Canada.

1 Sobel JD. Vulvovaginal candidiasis-What we do and do not know. Ann Intern Med 1984;101:390-2.

2 Witkin SS, Yu IR, Ledger WJ. Inhibition of Candida albicans-induced lymphocyte proliferation by lymphocytes and sera from women with recurrent vaginitis. $A m \mathcal{F}$ Obstet Gynecol 1983;147:809-11.
3 Hurowitz BJ, Edelstein SW, Lippman L. Sexual transmission of Candida. Obstet Gynecol 1987;69:883-6.

4 Miles MR, Olsen L, Rogers A. Recurrent vaginal candidiasis; Importance of an Intestinal reservoir. $\mathcal{F} A M A$ 1977;238:1836-7.

5 Davidson B, Mould RF. Recurrent genital candidosis in women and the effect of intermittent prophylactic treatwomen and the effect of intermittent prop

6 Sobel JD. Recurrent vulvovaginal candidiasis. A prospective study of the efficacy of maintenance ketoconazole therapy. $N$ Engl $f$ Med 1986;1313:1455-8.

7 Van Cutsem J, Van Gerven F, Janssen PAJ. Activity of orally, topically and parenterally administered itraconazole in the treatment of superficial and deep mycoses: Anima Models. Rev Infect Dis 1987;9 Suppl I. S15-S32.

8 Silva Cruz A, Andrade L, Oliveira JE et al. An open assessment of three different regimens of itraconazole for the management of vaginal candidosis. Phar macotherapeutica 1988;5:189-92.

9 Van Cutsem J, Janssen PAJ. Les azoles antifongique. Med Malad Infect 1984;14:510.

10 Cauwenbergh G. Itraconazole: The first orally active antifungal for single-day treatment of vaginal candidosis. Curr Ther Res 1987;41:210-4.

11 Hardin TC, Graybill JR, Fetchick R, Woestenborghs R Rinaldi MG, Kuhn J. Pharmacokinetics of Itraconazole following oral administration to normal volunteers. Antimicrob Agents Chemother 1988;32:1310-3.

12 Larosa E, Cauwenberg G, Cilli P, Woestenborghs R Heykants J. Itraconazole pharmacokinetics in the female genital tract: Plasma and tissue levels in patients undergoing hysterectomy after a single dose of $200 \mathrm{mg}$ itraconazole. Eur 7 Obstet Gynecol Reprod Biol 1986;23:85-9.

13 Schafer-Korting M, Korting HC, Lukacs A, Hey Kants Behrendt H. Levels of itraconazole in skin blister fluid Behrendt $H$. Levels of itraconazole in skin blister fluid tion. F Am Acad Dermatol 1990;22:211-5.

14 Lewis JH, Zimmerman HJ, Benson GD, Ishak KG. Hepatic injury associated with ketoconazole therapy; analysis of 33 cases. Gastroenterology 1984;86:503-13. 